\title{
BMJ Global Health Prioritising the care of critically ill children: a pilot study using SCREEN reduces clinic waiting times
}

\author{
Bhakti Hansoti, ${ }^{1}$ Mohammed Dalwai, ${ }^{2}$ Joanne Katz, ${ }^{1}$ Martin Kidd, ${ }^{3}$ \\ Ian Maconochie, ${ }^{4}$ Alain Labrique, ${ }^{1}$ Lee Wallis ${ }^{2}$
}

To cite: Hansoti B, Dalwai M, Katz J, et al. Prioritising the care of critically ill children: a pilot study using SCREEN reduces clinic waiting times. BMJ Global Health 2016;1: e000036. doi:10.1136/ bmjgh-2016-000036

Received 2 February 2016 Revised 21 May 2016 Accepted 23 May 2016

\section{(a) CrossMark}

${ }^{1}$ Johns Hopkins University, Baltimore, Maryland, USA ${ }^{2}$ Department of Surgery, University of Cape Town, Cape Town, South Africa ${ }^{3}$ Stellenbosch University, Cape Town, South Africa ${ }^{4}$ St Mary's Hospital, Imperial College, London, UK

Correspondence to Dr Bhakti Hansoti; bhansot1@jhmi.edu

\section{ABSTRACT}

Objective: In low-resource settings, childhood mortality secondary to delays in triage and treatment remains high. This paper seeks to evaluate the impact of the novel Sick Children Require Emergency Evaluation Now (SCREEN) tool on the waiting times of critically ill children who present for care to primary healthcare clinics in Cape Town, South Africa.

Methods: We used a pre/postevaluation study design to calculate the median waiting times of all children who presented to four randomly chosen clinics for 5 days before, and 5 days after, the implementation of SCREEN.

Findings: The SCREEN programme resulted in statistical and clinically significant reductions in waiting times for children with critical illness to see a professional nurse (2 hours $45 \mathrm{~min}$ to 1 hour $12 \mathrm{~min}$; $\mathrm{p}<0.001)$. There was also a statistically significant reduction in the proportion of children who left without being seen by a professional nurse $(25.8 \%$ to $18.48 \%$; $p<0.001$ ).

Conclusions: SCREEN is a novel programme that uses readily available laypersons, trained to make a subjective assessment of children arriving at primary healthcare centres, and provides a low cost, simple methodology to prioritise children and reduce waiting times in low-resource healthcare clinics.

\section{INTRODUCTION}

Reducing child mortality worldwide remains a challenge. ${ }^{1}$ In 2013, 6.3 million children under the age of 5 years died, and it is estimated that $70 \%$ of these deaths were due to conditions that can be prevented, or treated, with timely access to simple, affordable interventions. ${ }^{2}$ Therefore, it is essential to implement strategies to combat delays in identification and treatment of critically ill children in low-resource settings.

In low income and middle income countries, children with critical illnesses are often initially brought to primary healthcare clinics (PHC) rather than hospitals. Such clinics see numerous patients with a wide range of illness severity. ${ }^{3}$ To guide the management of children

\section{Key questions}

What is already known about this topic?

- Primary healthcare clinics (PHC) that see large volumes of patients with a wide range of illness severity in low-resource settings.

- Waiting times in PHC remain high due to large volumes of patients and a lack of trained healthcare providers.

- Childhood mortality remains high in PHC in lowresource settings due to delays in the identification and treatment of children with critical illness.

\section{What are the new findings?}

- Sick Children Require Emergency Evaluation Now (SCREEN) is a novel tool for low-resource PHC that is quickly implemented by laypersons to identify children with critical illnesses.

- The implementation of SCREEN significantly reduced waiting times for critically ill children.

- SCREEN improved the efficiency of the clinic and had a positive impact on the left without being seen rate.

\section{Recommendations for policy}

- SCREEN provides a low cost, easily implementable, simple methodology to prioritise children and reduce waiting times in lowresource healthcare clinics.

presenting to the clinic with an acute illness, a cohesive standardised approach is required. To address this need, the WHO and UNICEF collaborated to develop the Integrated Management of Childhood Illnesses (IMCI) approach, which was launched in $1996 .^{3}$ The goal of IMCI is to improve the performance of healthcare workers (HCWs), by combining lessons from disease-specific control programmes to develop a single efficient and effective syndrome-based approach. ${ }^{4}$ The HCWs use syndrome-centred algorithms to triage children, and deliver care, directed by well-defined management plans based on the child's severity of illness. 
Since 1996, IMCI has been widely implemented in over 80 countries. ${ }^{5}$ However, there are significant limitations: the case management process requires a trained HCW (professional nurse equivalent), and takes 5-7 min to complete. Owing to an inadequate number of trained HCWs, and large numbers of children presenting to PHC, the waiting times remain long, even after a child has been successfully brought to a facility. ${ }^{6}$ For critically ill children, the waiting time of several hours, without evaluation and the initiation of treatment, can be fatal to a child's chance of survival.

A key to reducing under five mortality is to implement a cost-effective programme that promptly identifies critically ill children, prioritises their care and thereby reduces waiting times. ${ }^{7}$ Our team worked together with local staff to develop a prioritisation tool to reduce these delays. We identified three groups of stakeholders: (1) healthcare providers at the PHCs, (2) experts in paediatric emergency and acute care; and (3) the executive healthcare management team of the Cape Town government. We developed the algorithm entitled Sick Children Require Emergency Evaluation Now (SCREEN). The screening tool was derived from the validated WHO IMCI danger signs. ${ }^{4}$ This new tool was then pilot-studied at a single PHC, and further refined using action research methodology which consisted of cycles of piloting the tool in a single clinic, observing its use, receiving feedback from the nursing staff and adjusting it. SCREEN is administered by laypersons, that is, queue marshals $(\mathrm{QM})$ at the point of entry to a clinic, and uses six simple questions to rapidly identify critically ill children and expedite their care:

1. Ask ... Is your child unable to drink or breastfeed?

2. Ask ... Is your child vomiting everything?

3. Ask... Has your child had convulsions during this illness?

4. Look...See if the child is lethargic or unconscious.

5 . Ask... Is your child $<2$ months old?

6. Ask... Have you been to a clinic/hospital in the past 2 days?

SCREEN training is provided by the City of Cape Town training division, and consists of a half-day review of IMCI danger signs with a half-day of in-clinic supervised practice.

The key to successful implementation and adoption of a new clinical intervention is the ability to improve individual patient outcomes without disrupting the clinical environment. ${ }^{8}$ The SCREEN programme was developed for a complex clinical environment where high volumes of patients, both sick and not sick, routinely present for care. Thus, it was important to determine the impact of SCREEN on waiting times for three groups of children at the PHC: critically ill, those with minor illnesses and healthy children who present for routine healthcare. An intervention for critically ill children (fewer than $10 \%$ of patients) that causes delays in the well population (60-70\% of patients) would potentially deter patients from attending the clinic. ${ }^{9}$ To evaluate the impact of SCREEN on waiting times, we measured the flow of all children who presented to clinics for care pre-SCREEN and post-SCREEN implementation.

\section{METHODS}

\section{Study site}

This study was conducted from 1 March to 1 September 2014 in PHCs in Cape Town, South Africa. PHCs provide care to all children within their catchment areas. The clinics see both sick children, a small percentage of whom are critically ill, and well children who present for routine immunisations, weighing and nutrition assessments. Clinics normally see patients from 8:00 to 16:00 from Monday to Friday.

Within each PHC, a nurse facility manager, who is a senior professional nurse (PN), organises and supervises care. Sick children are attended to by a combination of PNs and enrolled nurses (ENs). The PNs, graduates of a 4-year nursing degree programme, who have received training in IMCI, complete the formal consultation for each sick child and decide the management plan. In contrast, ENs are graduates of a 2-year nursing degree programme; they collect basic vital signs and perform weight assessment and basic diagnosis using IMCI. Each child must be seen by an EN prior to a PN evaluation. Each clinic has one or two ENs, and one to four PNs at any given time, depending on the patient load of the clinic.

\section{Sampling and study design}

In this pilot study, four clinics out of the 120 PHCs in the City of Cape Town were randomly recruited for enrolment in this study. Owing to the heterogeneous nature of the clinics, the unique clinic layout at each site and staffing dynamics, we used a pre/postevaluation study design. Included in the analysis were all children who presented to the clinic for five randomly chosen consecutive days (ie, 1 week), pre and post-SCREEN implementation. The sampling time frame was limited by funding resources.

All four clinics provide care to adults and children. In all clinics, children queued separately and were enrolled prior to entering the clinic, while study coordinators asked parents to put a QR code sticker on each child prior to entry. At all clinics, patients were first scanned at entry into the clinic. Written consent was not required by our protocol. All parents were informed that we were using the QR codes to track the flow of patients; compliance with placing the sticker on the child was given as consent to participate.

\section{Data capture}

To ensure accurate capture of data, patient tracking software designed by The Open Medicine Project South Africa (TOMPSA) was created specifically for this study. Each child who presented to the clinic was allocated a randomly generated four-digit number, encoded in a quick response $(\mathrm{QR})$ code sticker that was placed on the 


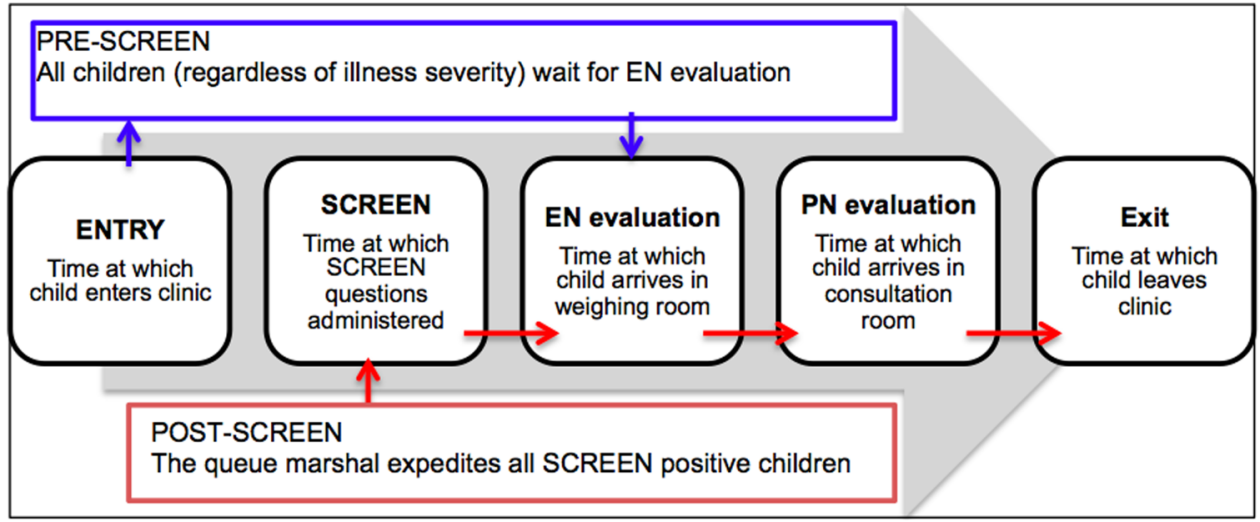

Figure 1 Five patient-tracking interaction points for electronic capture. EN, enrolled nurse; SCREEN, Sick Children Require Emergency Evaluation Now.

child's clothing outside the clinic (before they entered the clinic and joined the waiting line). A QR code is a machine-readable code consisting of an array of black and white squares.

Each member of staff was given an Android smartphone with which to track patient flow. A custom-coded application captured the $\mathrm{QR}$ code and transmitted the 'time of scanning' to a website (specifically designed data capture system). If data connectivity was unavailable, the captured data were stored on the phone and uploaded subsequently. Using this system, five time points were recorded: (1) when the child entered the clinic, (2) the time at which SCREEN are asked, (3) was seen by an EN in the weighing room, (4) was seen by a $\mathrm{PN}$ in the treatment room and (5) left the clinic (figure 1).

At the time of scanning the QR codes, nurses (time points (3) and (4)) were asked to record the IMCI category (red/yellow/green) to which they considered the child to belong. The IMCI categories (table 1) were used to delineate each child's severity of illness, as this is currently the standard accepted practice in the PHC. For each child, the IMCI category assigned by the PN was used for analysis; if this was unavailable, the EN assigned category was used.

\section{Quality assurance}

To ensure that the electronic data collection was accurate, a subset of data was collected manually for comparison. A research assistant interviewed a sample of $10 \%$ of patients exiting the clinic (achieved by interviewing all

Table $1 \mathrm{IMCl}$ categories and their definitions and implications

\begin{tabular}{lll}
\hline Category & Definition & Implication \\
\hline Red & $\begin{array}{l}\text { Critically ill/ } \\
\text { life-threatening illness }\end{array}$ & $\begin{array}{l}\text { Transfer immediately } \\
\text { for treatment }\end{array}$ \\
Yellow & Sick & $\begin{array}{l}\text { Treatment and } \\
\text { observation in clinic }\end{array}$ \\
Green & Well & No active treatment \\
\hline
\end{tabular}

$\mathrm{IMCl}$, Integrated Management of Childhood Illnesses. patients who attended the clinic for one of the 5 days during data collection). The adult accompanying the child was asked to recall at what time, and by whom, they were seen in the clinic. This information was compared to the data captured electronically using the $\mathrm{QR}$ code system. An encounter was deemed concordant if there was agreement between the electronic and manual data on the hour and who saw the child. A priori, a $>90 \%$ concordance was decided on as a threshold for inclusion in the analysis. All clinics were able to meet this requirement.

\section{Outcome measures and data analysis}

The primary outcome measure was the mean time from entry into the clinic to the first nursing encounter, for each clinic, preintervention and postintervention, for each of the three IMCI categories. We also calculated the mean throughput time (entry to exit). Another key outcome was the number of children who left without being seen (LWBS) by an EN or PN.

Statistical analysis was carried out using two-way analysis of variance with SCREEN (preimplementation and postimplementation) and acuity (IMCI red, yellow, green) as the two factors. Post hoc tests were performed using Fisher's least significant difference testing. The number of children that LWBS pre-SCREEN and post-SCREEN was compared using cross tabulation and $\chi^{2}$ tests. A $5 \%$ significance level was used as the threshold for determining significant differences.

\section{Ethics approval}

Verbal consent was requested from all parents to allow their child to be tagged by a QR code, and for the code to be scanned as various healthcare providers saw the child in the clinic.

\section{RESULTS \\ Overall}

A total of 3064 children were enrolled in the study. No severity of illness was assigned for $3 \%$ of children as they 
left prior to evaluation by a PN or EN. Eighty-five per cent of patients were IMCI green $(\mathrm{n}=2601), 9.5 \%$ yellow $(\mathrm{n}=288)$ and $2.5 \%$ red $(\mathrm{n}=81)$. The remaining 94 patients $(3 \%)$ left without being assigned a severity category. All IMCI red patients received evaluation from a PN. Eight per cent $(23 / 288)$ of IMCI yellow patients and 22\% (571/2601) of IMCI green patients were seen only by an EN.

A similar number of children presented to all four clinics. Clinic 3 saw the highest volume of children at a total of 778 and clinic 4 saw the lower number of children at 624. The number of SCREEN positive children during the 5-day assessment postimplementation varied from $40(13 \%)$ in clinic 2 to $26(7 \%)$ in clinic 3. In all four clinics, all 'IMCI Red' children were correctly identified as 'SCREEN-positive' (table 2).

\section{Waiting times analysis}

Implementation of SCREEN was associated with a significant reduction in the mean time that critically ill (IMCI red) children waited to see an EN and a PN at clinics 2, 3 and 4 (table 3). The greatest reduction in waiting times for these children was at clinic 4, in which the mean wait time to see an EN was reduced from 2 hours $24 \mathrm{~min}$ to $20 \mathrm{~min}$, and the mean wait time to see a PN was reduced from 3 hours 13 min to 1 hour 4 min.

\section{Clinic flow}

After implementation of SCREEN, there was a statistically significant decrease in the mean amount of time children spent in the clinic, from entry to exit in clinics 1,3 and 4 (table 3). In contrast, at clinic 2, after implementation of SCREEN, there was a slight-but not statistically significant-increase in the mean amount of time children spent in the clinic.

\section{Left without being seen}

After implementation of SCREEN, there was a statistically significant reduction in the proportion of children who LWBS by a PN in clinics 2 and 4 (table 4). There were also decreases in clinics 1 and 3, though these did not reach statistical significance.

\section{Aggregate analysis}

Data for all four clinics combined showed that implementation of the SCREEN programme resulted in statistically, and clinically significant, reductions in waiting times for children with critical illness (IMCI Red), and those that required treatment in the clinic (IMCI Yellow) (table 5); there was no impact on the waiting time for well children (IMCI green). There was also a statistically and clinically significant reduction in the proportion of children that LWBS by a PN (table 5). There was no impact of SCREEN on the proportion of children that LWBS by an EN.

\section{DISCUSSION}

\section{Waiting times}

This pilot study demonstrates that implementation of the SCREEN programme in low-resource primary healthcare clinics can significantly reduce the waiting times for critically ill children. This study enrolled 3064 children at four different clinical sites, and the significant reduction in waiting times, from time of entry to seeing a healthcare provider, was a consistent finding in all four of the clinical sites.

Each clinic provided care to $~ 350-400$ patients per week ( 75 children per day). Most clinics had only one EN, who was the first point of care for all children, and who was responsible for weighing the child, collecting vital signs and identifying the child as critically ill using IMCI. In this clinic model, the high volumes of children presenting for care, and the time-consuming initial evaluation probably contributed to the long wait times and high LWBS rates. Our new SCREEN programme performed well despite the high patient volumes and lack of qualified healthcare providers in the enrolled clinics. This is most likely due to the design of SCREEN, which task shifts screening to a layperson and requires only a subjective assessment that takes less than a minute per child to complete.

All IMCI Red patients demonstrated significant reductions (over 1 hour) in waiting times for initial healthcare provider evaluation. Critically ill children often present with haemodynamic instability and time-sensitive illness that can be reversed by timely supportive management. In these children, 1 hour can have a large impact on survival and be the difference between life and death.

We had expected that only the care of critically ill children would be expedited (given the sensitivity of SCREEN), and that there may even be a negative impact on waiting times for all other children (IMCI yellow and IMCI green) who presented to the clinic. However, our study showed a decrease in waiting times for all children who presented to the clinic (even those presenting for routine well child checks, vaccinations and deworming). This secondary, and positive, effect is most likely due to the fact that SCREEN streamlined the flow of critically ill children, and thus decreased interruptions to the care of the remaining children. For example, prior to SCREEN, critically ill children would wait in line to be seen (sometimes for hours), until their first interaction with a healthcare provider. ${ }^{6}$ Throughout the day, PNs would have to divert their focus to provide life-saving treatment and arrange transfer of care. However, once SCREEN was implemented, most critically ill children would be identified early in the morning, given that over $50 \%$ of patients arrive before the clinics open, and their care and transfer could be coordinated by a single $\mathrm{PN}$, thus allowing the remainder of the clinic to continue undisturbed. Anecdotally we observed that multiple critically unwell children were pooled into a single ambulance to be transferred to a higher level of care. In a resource-limited setting, this approach is an efficient 
Table 2 Number and percentage of children presenting by IMCl acuity category, at each of four clinics, preimplmentation and postimplementation of SCREEN

\begin{tabular}{|c|c|c|c|c|c|c|c|c|}
\hline \multirow[b]{2}{*}{ Acuity } & \multicolumn{2}{|l|}{ Clinic 1} & \multicolumn{2}{|l|}{ Clinic 2} & \multicolumn{2}{|l|}{ Clinic 3} & \multicolumn{2}{|l|}{ Clinic 4} \\
\hline & Pre-SCREEN & Post-SCREEN & Pre-SCREEN & Post-SCREEN & Pre-SCREEN & Post-SCREEN & Pre-SCREEN & Post-SCREEN \\
\hline Red & 5 & $16^{*}$ & 13 & $13^{*}$ & 5 & $10^{*}$ & 10 & $8^{*}$ \\
\hline Per cent & 1.22 & 4.01 & 3.06 & 4.30 & 1.13 & 2.97 & 3.09 & 2.74 \\
\hline Yellow & 51 & 27 & 20 & 28 & 36 & 29 & 63 & 32 \\
\hline Per cent & 12.44 & 6.77 & 4.71 & 9.27 & 8.16 & 8.61 & 19.44 & 10.96 \\
\hline Green & 354 & 356 & 392 & 274 & 400 & 308 & 251 & 260 \\
\hline Per cent & 86.34 & 89.22 & 92.24 & 90.73 & 90.71 & 91.39 & 77.47 & 89.04 \\
\hline TOTAL & 410 & 399 & 425 & 302 & 441 & 337 & 324 & 292 \\
\hline
\end{tabular}

*All children were also correctly identified as SCREEN positive by the QM.

IMCI, Integrated Management of Childhood Illnesses; SCREEN, Sick Children Require Emergency Evaluation Now; QM, queue marshals.

Table 3 Median time to evaluation by nursing staff (EN, enrolled nurse; PN, professional nurse) by acuity and clinic, preimplementation and postimplementation of SCREEN

\begin{tabular}{|c|c|c|c|c|c|c|c|c|}
\hline \multirow[b]{2}{*}{ Acuity } & \multicolumn{2}{|l|}{ Clinic 1} & \multicolumn{2}{|l|}{ Clinic 2} & \multicolumn{2}{|l|}{ Clinic 3} & \multicolumn{2}{|l|}{ Clinic 4} \\
\hline & Pre-SCREEN & Post-SCREEN & Pre-SCREEN & Post-SCREEN & Pre-SCREEN & Post-SCREEN & Pre-SCREEN & Post-SCREEN \\
\hline EN-Red & $0: 54 †$ & $0: 31 \dagger$ & $1: 00$ & $0: 16^{\star \star \star}$ & $1: 11$ & $0: 12^{\star \star \star}$ & $2: 28$ & $0: 20^{\star \star *}$ \\
\hline PN-Red & $2: 29 \dagger$ & $1: 31 \dagger$ & $1: 29$ & $0: 58^{\star \star}$ & $1: 44$ & $1: 09^{\star \star}$ & $3: 13$ & $1: 04^{\star \star}$ \\
\hline EN-Yellow & $1: 33$ & $0: 51^{\star \star \star}$ & $0: 47$ & $0: 20^{\star * *}$ & $1: 20$ & $1: 13$ & 3:01 & $1: 27^{\star \star \star}$ \\
\hline PN-Yellow & $3: 25$ & $2: 42^{\star \star}$ & $2: 07$ & $2: 08$ & $2: 07$ & $2: 31$ & $4: 20$ & $2: 50^{\star \star}$ \\
\hline EN-Green & $1: 43$ & $1: 22^{\star \star \star}$ & $1: 05$ & $0: 47^{\star \star \star}$ & $1: 55$ & $2: 02$ & $2: 38$ & $2: 29$ \\
\hline PN-Green & $3: 00$ & $2: 41^{\star \star \star}$ & $1: 19$ & $1: 28$ & $2: 54$ & $2: 46$ & $3: 52$ & $3: 49$ \\
\hline EXIT & $3: 19$ & $3: 00^{\star \star \star}$ & $2: 05$ & $2: 23$ & $3: 14$ & $2: 59^{*}$ & $4: 41$ & $4: 31^{*}$ \\
\hline
\end{tabular}

Bold typeface indicates significance.

Times are given in hrs:mins.

${ }^{*}$ Statistical significance ${ }^{*} \mathrm{p}<0.1,{ }^{* *} \mathrm{p}<0.05,{ }^{* * *} \mathrm{p}<0.01$.

†Decrease in waiting times may not have reached statistical significance secondary to low volume of patients.

SCREEN, Sick Children Require Emergency Evaluation Now.

Table 4 Number and percentage of patients who LWBS, by nursing level and clinic, preimplementation and postimplementation of SCREEN

\begin{tabular}{|c|c|c|c|c|c|c|c|c|}
\hline & \multicolumn{2}{|l|}{ Clinic 1} & \multicolumn{2}{|l|}{ Clinic 2* } & \multicolumn{2}{|l|}{ Clinic 3} & \multicolumn{2}{|l|}{ Clinic $4^{\star}$} \\
\hline & $\begin{array}{l}\text { Pre-SCREEN } \\
(\%)\end{array}$ & $\begin{array}{l}\text { Post-SCREEN } \\
\text { (\%) }\end{array}$ & $\begin{array}{l}\text { Pre-SCREEN } \\
(\%)\end{array}$ & $\begin{array}{l}\text { Post-SCREEN } \\
\text { (\%) }\end{array}$ & $\begin{array}{l}\text { Pre-SCREEN } \\
(\%)\end{array}$ & $\begin{array}{l}\text { Post-SCREEN } \\
(\%)\end{array}$ & $\begin{array}{l}\text { Pre-SCREEN } \\
(\%)\end{array}$ & $\begin{array}{l}\text { Post-SCREEN } \\
(\%)\end{array}$ \\
\hline Enrolled Nurse -EN & $\begin{array}{l}17 \\
4.01\end{array}$ & $5.1^{21}$ & $\begin{array}{l}8 \\
1.9\end{array}$ & $\begin{array}{l}7 \\
2.1\end{array}$ & $\begin{array}{l}17 \\
3.74\end{array}$ & $\begin{array}{l}15 \\
4.19\end{array}$ & $\begin{array}{l}33 \\
9.4\end{array}$ & $\begin{array}{l}20 \\
6.27\end{array}$ \\
\hline $\begin{array}{l}\text { Professional } \\
\text { Nurse-PN }\end{array}$ & $\begin{array}{l}68 \\
16\end{array}$ & 5513.5 & 17641 & $\begin{array}{l}97 \\
30\end{array}$ & 10423 & $\begin{array}{l}72 \\
20\end{array}$ & $\begin{array}{l}80 \\
23\end{array}$ & $\begin{array}{l}36 \\
11\end{array}$ \\
\hline
\end{tabular}

*Shows a statistically significant $(\mathrm{p}<0.05)$ decrease in the proportion of patients who LWBS by a professional nurse (PN).

LWBS, left without being seen; SCREEN, Sick Children Require Emergency Evaluation Now. 
Table 5 Aggregate analysis of waiting times and LWBS counts, by nursing level, pre-SCREEN and post-SCREEN implementation

\begin{tabular}{lllll}
\hline Waiting times & Acuity* & $\begin{array}{l}\text { Pre-SCREEN } \\
\text { h:min }\end{array}$ & $\begin{array}{l}\text { Post-SCREEN } \\
\text { h:min }\end{array}$ & $\begin{array}{r}\text { p Value } \\
\hline\end{array}$ \\
\hline EN-RED & $1: 35$ & $\mathbf{0 : 2 0}$ & $<\mathbf{0 . 0 0 1}$ \\
& PN-RED & $2: 45$ & $\mathbf{1 : 1 2}$ & $<0.001$ \\
& EN-YELLOW & $1: 58$ & $\mathbf{0 : 5 9}$ & $<\mathbf{0 . 0 0 1}$ \\
& PN-YELLOW & $3: 14$ & $\mathbf{2 : 2 9}$ & $<\mathbf{0 . 0 0 1}$ \\
& EN-GREEN & $1: 45$ & $1: 37$ & 0.01 \\
LWBS & PN-GREEN & $3: 00$ & $2: 56$ & 0.337 \\
& & Counts, \% & Counts, \% & 0.948 \\
& EN & $75,4.53$ & $63,4.48$ & $<\mathbf{0 . 0 0 1}$ \\
\hline
\end{tabular}

Bold typeface indicates significance.

${ }^{*}$ Acuity is determined by IMCl category assigned by the professional nurse (PN); if this is unavailable, enrolled nurse (EN) assignment was used. $\mathrm{IMCl}$, Integrated Management of Childhood Illnesses; LWBS, left without being seen; SCREEN, Sick Children Require Emergency Evaluation Now.

use of the limited prehospital care teams available to the clinic.

It is also likely that the positive impact of SCREEN on waiting for IMCI yellow and green children may be secondary to the fact that some of these children will have most likely been expedited after incorrectly being categorised as SCREEN positive. In this study, we did not record the proportion of patients identified using SCREEN, merely recording how implementing a screening intervention impacted patient flow.

Unfortunately, despite the significant reduction in waiting times following implementation of SCREEN, the mean waiting times for a PN to see a critically ill child remained disturbingly high (58 min -1 hour $31 \mathrm{~min}$ ). By definition, these children require immediate life-saving interventions and immediate transfer of care. Many would argue that an hour's wait is still too long. ${ }^{10} 11$ Other operational issues continue to contribute to the delay in care for critically ill children. First, despite the QM identifying the child as critically ill, most clinics required that the child be taken to the EN for vital signs, weighing and documentation of chief symptom, prior to being seen by a PN. Second, most PNs would not agree to see a child without their folder; thus, delays in searching for the patient's clinic folder could delay care. Third, despite the QM identifying the child as SCREEN positive, there was sometimes resistance from the ENs and PNs in prioritising children, due to the belief that all children should await their turn, and/or the EN or PN did not trust the assessment of an untrained layperson. These systematic and behavioural issues need to be better defined by an implementation study, ${ }^{12} 13$ and could be the basis for further interventions such as providing IMCI training to the lay 'queue marshals' as well so that they may be able to better advocate patients who were SCREEN positive.

\section{Left without being seen}

After implementation of SCREEN, the percentage of children who left the clinics without being seen by a healthcare provider decreased at each clinic, with significant decreases at two of the four clinics. The number of patients who did not see a PN pre-SCREEN and post-SCREEN reduced by over $25 \%$ (25.8\% to $18.48 \%$, respectively). We attribute this improvement to having a clinic representative (the QM) engage the parents early in their child's presentation to the clinic, an action which may have assured them that the clinic was invested in the care of their child. Furthermore, this initial human interaction also allowed parents to ask questions about the system and waiting times, which may have made them more likely to wait for their child's evaluation than in the absence of such information. ${ }^{14} 15$

Clinic 2 had the most significant drop in the LWBS rate; however, we note that this may have resulted in clinic 2 having a slight increase in overall waiting times. This increase was most likely due to the fact that more children were staying to complete a full evaluation (ie, were seen by an EN and $\mathrm{PN}$ ) before leaving the clinic.

\section{Implementation and future work}

This study evaluates a simple intervention in low resource clinics to prioritise care. The current cost to each clinic per QM is averaged at $\$ 10-15$ per day. The QM workforce is readily available given that no prior qualifications are required for this job. In addition, the training time for QMs is only 1 day. However, when expanded to the City of Cape Town, if one would propose QM coverage in all of the 120 clinics year round, the forecasted cost is in excessive of $\$ 350000$ per year. In resource-limited settings, where financial constraints hinder the adoption of most healthcare interventions, this may be cost prohibitive. Anecdotally, in the City of Cape Town, we have found that some of the clinics opted to expand the screening role to security guards and record room staff as opposed to hiring QMs.

Overall, in busy clinics where an additional member of staff is required, this appears be a cost-effective solution that improves patient flow and reduces life-threatening delays in 
the care for critically ill children. Our study only evaluates the impact of SCREEN when a dedicated provider uses the tool. Further study is required to evaluate how the flow of children will be impacted if screening is added as a secondary role for security guards and record staff.

Delays in care for critically ill children that exceed over 1 hour are unacceptable and thus must be addressed. SCREEN provides a simple solution to significantly reduce waiting times in the clinic setting by task shifting the role of identification of critically ill patients to laypersons. Large volume scale-up will require further study on how to optimise implementation so that waiting times for critically ill children are $<20 \mathrm{~min}$. Currently, the City of Cape Town has adopted the SCREEN tool and QMs as standard of care in all of its high volume clinics.

\section{LIMITATIONS}

The biggest challenge with research involving direct observation and performance measurements is the well described 'Hawthorne Effect'. To minimise the incentive for staff to artificially scan/see children quicker than normal, we (1) recruited local study staff and avoided the presence of 'foreign' researchers at the study site, (2) staff were informed that data captured is anonymised, and thus individual performances cannot be ascertained and (3) that the individual's data will not be shared with clinic leadership.

While the novel QR code and Android phone-based patient-tracking system designed for this study allowed the accurate tracking of children in the clinics, there may have been some difficulties in their use. Although all providers were asked to scan the $\mathrm{QR}$ codes at the start of their interaction with the child, it is possible that this was forgotten, or was performed inconsistently, thereby biasing the data capture. However, this limitation was addressed by implementing a quality assurance protocol where $10 \%$ of clinic attendees were interviewed. We found that during this audit, each of the clinical sites demonstrated a $>90 \%$ concordance of the electronically collected data compared to self-reporting. Thus, no data were excluded from this study. To retain a normal workflow pattern, by avoiding the interruption caused by scanning the QR codes, a simpler approach may involve the use of radio-frequency identification tags, in which there is electronic capture the instant a patient reaches a specific location. However, this approach was beyond the constraints of the study budget, and would present difficulties in a small, resource poor clinic environment where patients often walk back and forth between several locations.

In addition, staffing variability at each of the clinics is most likely an important confounder for the presented data. Staffing levels varied on a daily basis depending on sick leave, staff's ability to get to work and the number of staff assigned to the paediatric workload. The latter was dependent on the volume of adult patients and if any additional special clinics (TB care, etc) were being run that day. In order to account for the daily variability, each clinic was sampled for 5 consecutive days.

\section{CONCLUSIONS}

SCREEN is a novel programme that task shifts the role of identifying and prioritising the care of critically ill children to laypersons using a subjective assessment tool. In our pilot study, the implementation of SCREEN reduced waiting times for all critically ill children, and had a positive impact on the LWBS rates in all clinics. Owing to a paucity of healthcare resources in low-resource settings worldwide, waiting times for critically ill children and downstream mortality remain unacceptably high. SCREEN provides a low cost, simple solution that may be implemented to meet this need. Further evaluation is necessary to inform scale-up and refinement of this novel intervention in clinical settings, beyond South Africa, where sick children present for care.

Handling editor Soumitra Bhuyan

Twitter Follow Bhakti Hansoti at @BhaktiHansoti

Acknowledgements The authors would like to thank the City of Cape Town Health Management Team and the division for training for assistance in the conduct of this study and in the development of SCREEN.

Contributors BH conceptualised the study, designed the methodology, collected the data, and wrote and edited the manuscript. MD and LW conceptualised the methodology, assisted with data acquisition, and assisted in editing the manuscript. JK and MK made contributions to the analysis and interpretation of data and also to drafting the work or revising it critically for important intellectual content. AL and IM made substantial contributions to the conception of the study and revising the manuscript for intellectual content. All authors made substantial contributions to the study, both to the drafting of the work and its critical revision for important intellectual content; AND have given final approval of the version to be published; AND are in agreement to be accountable for all aspects of the work in ensuring that questions related to the accuracy or integrity of any part of the work are appropriately investigated and resolved.

Funding $\mathrm{BH}$ received funding from the Global Health Program for Fellows and Scholars (Global Health Fellows) (R25) (RFA-TW-11-001). Additional financial support received from the JHU Global mHealth Initiative.

Competing interests None declared.

Ethics approval IRB approval was received from the primary authors' home institution, Johns Hopkins University in Baltimore, USA, and the host institution, the University of Cape Town, South Africa.

Provenance and peer review Not commissioned; externally peer reviewed.

Data sharing statement Non-identified data (ie, without protected health information) will be made available in an excel spreadsheet by request to the corresponding author. The following data set is available for all paediatric patients time they entered the clinic, time at which they were seen by an EN/ $\mathrm{PN}$, time of treatment and time at which the child exited the clinic.

Open Access This is an Open Access article distributed in accordance with the Creative Commons Attribution Non Commercial (CC BY-NC 4.0) license, which permits others to distribute, remix, adapt, build upon this work noncommercially, and license their derivative works on different terms, provided the original work is properly cited and the use is non-commercial. See: http:// creativecommons.org/licenses/by-nc/4.0/

\section{REFERENCES}

1. Black RE, Morris SS, Bryce J. Where and why are 10 million children dying every year? Lancet 2003;361:2226-34. 
2. UNICEF. UNICEF Data: Monitoring the Situation of Children and Women. The State of the World's Children Report. 2015.

3. Tulloch J. Integrated approach to child health in developing countries. Lancet 1999;354(Suppl 2):SIl16-20.

4. El Arifeen S, Blum LS, Hoque DM, et al. Integrated Management of Childhood Illness (IMCI) in Bangladesh: early findings from a cluster-randomised study. Lancet 2004:364:1595-602.

5. Bryce J, Victora CG, Habicht JP, et al. The multi-country evaluation of the integrated management of childhood illness strategy: lessons for the evaluation of public health interventions. Am J Public Health 2004;94:406-15.

6. Hodkinson P, Argent A, Wallis L, et al. Pathways to care for critically ill or injured children: a cohort study from first presentation to healthcare services through to admission to intensive care or death. PLOS ONE 2016:11:e0145473.

7. Nolan T, Angos P, Cunha AJ, et al. Quality of hospital care for seriously ill children in less-developed countries. Lancet 2001;357:106-10.

8. Kitson A, Harvey G, McCormack B. Enabling the implementation of evidence based practice: a conceptual framework. Qual Healthcare 1998;7:149-58.
9. Cabana MD, Rand CS, Powe NR, et al. Why don't physicians follow clinical practice guidelines? A framework for improvement. JAMA 1999;282:1458-65.

10. Razzak JA, Kellermann AL. Emergency medical care in developing countries: is it worthwhile? Bull World Health Organ 2002;80:900-5.

11. Beckmann U, Gillies DM, Berenholtz SM, et al. Incidents relating to the intra-hospital transfer of critically ill patients. Intensive Care Med 2004;30:1579-85.

12. French SD, Green SE, O'Connor DA, et al. Developing theory-informed behaviour change interventions to implement evidence into practice: a systematic approach using the Theoretical Domains Framework. Implement Sci 2012;7:38.

13. Davis DA, Taylor-Vaisey A. Translating guidelines into practice: a systematic review of theoretic concepts, practical experience and research evidence in the adoption of clinical practice guidelines. CMAJ 1997;157:408-16.

14. Wiler JL, Gentle C, Halfpenny JM, et al. Optimizing emergency department front-end operations. Ann Emerg Med 2010;55:142-60.e1.

15. Fernandes CM, Price A, Christenson JM. Does reduced length of stay decrease the number of emergency department patients who leave without seeing a physician? J Emerg Med 1997;15:397-9. 